\title{
Concomitant cetuximab and radiation therapy: A possible promising strategy for locally advanced inoperable non-melanoma skin carcinomas (Review)
}

\author{
GIUSEPPINA DELLA VITTORIA SCARPATI ${ }^{1}$, FRANCESCO PERRI ${ }^{2}$, SALVATORE PISCONTI $^{2}$, \\ GIUSEPPE COSTA $^{3}$, FILIPPO RICCIARDIELLO ${ }^{4}$, SALVATORE DEL PRETE ${ }^{5}$, ALBERTO NAPOLITANO ${ }^{4}$, \\ MARCO CARRATURO ${ }^{6}$, SALVATORE MAZZONE ${ }^{3}$ and RAFFAELE ADDEO ${ }^{5}$
}

\begin{abstract}
${ }^{1}$ D.A.I. Diagnostica Morfologica e Funzionale, Radioterapia e Medicina Legale, Università degli Studi di Napoli 'Ferderico II', I-80138 Napoli; ${ }^{2}$ Unità Operativa di Oncologia, Ospedale 'San Giuseppe Moscati' di Taranto, I-74100 Taranto; ${ }^{3}$ Dipartimento di Salute Mentale Fisica e Medicina Preventiva, SUN Napoli, I-80138 Napoli; ${ }^{4}$ ORL Unit, AORN Cardarelli, I-80131 Napoli;

${ }^{5}$ Oncology Unit, 'San Giovanni di Dio’ Hospital, A.S.L. Napoli 2 Nord, I-80027 Frattamaggiore (NA);

${ }^{6}$ Radiotherapy Unit, Medicina Futura, I-80035 Acerra, Italy
\end{abstract}

Received August 24, 2015; Accepted December 17, 2015

DOI: $10.3892 / \mathrm{mco} .2016 .746$

\begin{abstract}
Non-melanoma skin cancers (NMSCs) include a heterogeneous group of malignancies arising from the epidermis, comprising squamous cell carcinoma (SCC), basal cell carcinoma (BCC), Merkel cell carcinoma and more rare entities, including malignant pilomatrixoma and sebaceous gland tumours. The treatment of early disease depends primarily on surgery. In addition, certain patients present with extensive local invasion or metastasis, which renders these tumours surgically unresectable. Improving the outcome of radiotherapy through the use of concurrent systemic therapy has been demonstrated in several locally advanced cancer-treatment paradigms. Recently, agents targeting the human epidermal growth factor receptor (EGFR) have exhibited a consolidated activity in phase II clinical trials and case series reports. Cetuximab is a monoclonal antibody that binds to and completely inhibits the EGFR, which has been revealed to be up-regulated in a variety of SCCs, including NMSCs. The present review aimed to summarize the role of anti-EGFR agents in the predominant types of NMSC, including SCC and BCC, and focuses on the cetuximab-based studies, highlighting the biological rationale of this therapeutic option. In addition, the importance of the association between cetuximab and radiotherapy for locally advanced NMSC is discussed.
\end{abstract}

Correspondence to: Dr Raffaele Addeo, Oncology Unit, 'San Giovanni di Dio’ Hospital, A.S.L. Napoli 2 Nord, Via Pirozzi 62, I-80027 Frattamaggiore (NA), Italy

E-mail: lelloaddeo@alice.it

Key words: non-melanoma skin cancers, radiotherapy, chemotherapy, cetuximab, EGFR, squamous cell carcinoma, basal cell carcinoma

\section{Contents}

1. Introduction

2. Biology of NMSC and the rationale for using targeted therapy

3. Locally advanced NMSCs: The role of cetuximab

4. Conclusion

\section{Introduction}

Basal cell carcinoma (BCC) and squamous cell carcinoma (SCC) represent the more frequently occurring non-melanoma skin cancers (NMSCs), which are a group of malignancies arising from the epidermis, also comprising Merkel cell carcinoma and more rare entities, including malignant pilomatrixoma and sebaceous gland tumours. These malignancies are particularly frequent in the United States and geographical areas close to the Equator, including Australia (1). BCC, which originates from the cells composing the basal layer of the epidermis, occurs more frequently than SCC; it also presents a less aggressive behaviour and an improved prognosis. Immunosuppression, sun exposure and certain genetic diseases (e.g., xeroderma pigmentosum and Gorlin syndrome) are the most highly acknowledged risk factors (2). Since the 1960s, the incidence of NMSC worldwide has markedly increased, perhaps due to the progressive decrease in the stratospheric ozone stratum, with a consequent increased exposure to ultraviolet (UV) rays (3).

Early BCC and SCC [T1, with no risk factors (defined as small lesion, G1-2, no perineural invasion, no immunosuppression, no recurrent lesion)], may be treated effectively with surgery alone. The most frequently used surgical technique is Mohs micrographic surgery, which consists of the removal and extemporaneous analysis of every skin stratum until disease-free margins are identified. Excision of lesions with postoperative assessment of their margins is also 
widely employed, being less expensive compared with Mohs surgery and equally as efficacious as far as small tumours are concerned (4). Skin tumours that are locally advanced and/or have a high risk of recurrence (comprising $\mathrm{T}>1$ mass, invasion of perineural spaces, poor differentiation grade and spread to lymph nodes) may be treated with Mohs surgery combined (or not) with lymph-node dissection and/or adjuvant radiotherapy and chemotherapy combined (chemoradiotherapy) $(5,6)$.

Locally advanced SCCs that are medically inoperable or surgically non-resectable have a poor prognosis, although occasionally they may be cured with radiotherapy alone, or with chemoradiotherapy. In clinical trials, the combination of cisplatin and radiotherapy has yielded an improved outcome compared with radiotherapy alone, and this combination is the standard of care for non-resectable or inoperable SCC and $\mathrm{BCC}$ (7). Recurrent or metastatic diseases, which are more commonly observed in SCC compared with BCC, have a grim prognosis, and often are treated with systemic therapy. Systemic therapies that have been used in advanced NMSC include cytotoxic chemotherapy, immunotherapy and molecularly targeted agents (8-11), including bleomycin, 5-fluorouracil, 13-cis-retinoic acid, cisplatin, doxorubicin, interferon- $\alpha 2 \mathrm{a}$, gefitinib, cetuximab, capecitabine and erlotinib.

\section{Biology of NMSC and the rationale for using targeted therapy}

UV light exerts a fundamental role in the initiation and promotion of the carcinogenesis of NMSC, provoking the accumulation of genetic changes, which alter several oncogene-mediated pathways and, ultimately, lead to a selective growth advantage (5). One of these disrupted pathways is the epidermal growth factor receptor (EGFR) pathway. EGFRs are a family of tyrosine kinase transmembrane receptors, which include four different proteins, namely EGFR (or HER-1), c-erBB2 (or HER-2), ErbB-3 (or HER-3) and ErbB-4 (or HER-4) (12,13). Activation of EGFR, which normally is exerted by several extracellular soluble ligands, including EGF, results in autophosphorylation of the receptor's intracellular domains. This autophosphorylation leads to the activation of downstream effectors, including Ras and PI3K (phosphoinositide 3-kinase). The Ras- and the PI3K-stimulated pathways are able to elicit cell proliferation, activation of angiogenesis and inhibition of apoptosis $(14,15)$. EGFR is normally expressed in human cells, but higher levels of expression have been identified in numerous malignancies, including NMSCs (16). In previous studies [e.g., $(17,18)]$, particularly those employing immunohistochemical staining, $290 \%$ of the incidences of SCC and $60 \%$ of BCCs exhibited an overexpression of EGFR. The overexpression of EGFR is associated with poor prognosis in solid tumours, particularly SCC of the head and neck, and the degree of EGFR expression may correlate with the response to radiation therapy $(19,20)$. A severe overexpression of EGFR may correspond with a wide expression of receptors on the cell surface, leading to a constitutive activation of the downstream effectors, with generation of a marked, proliferative ligand-mediated signal. In fact, a specific clinical study has demonstrated a positive correlation between the intensity of EGFR expression and the nuclear proliferative index, $\mathrm{Ki}-67$, in head-and-neck SCC (21). Tumour cells increase their proliferative activity, and the repopulation effect derived from this mechanism may counteract the effects of radiotherapy $(22,23)$. The predominant EGFR downstream effector is the G-protein, Ras. Ras is tethered to cell membranes, and is activated by tyrosine kinase receptors, including EGFR (15). In several solid tumours, a DNA mutation of the ras gene is present, and this often leads to constitutive activation of Ras protein. This last step often leads to resistance to EGFR inhibitor drugs, including cetuximab, panitumumab, gefitinib and erlotinib. The percentage of ras mutations in NMSC widely varies: The values reported in clinical studies can range from 2 to $22 \%$ (24-26).

BCCs also express EGFR, but the key pathology in BCC is an aberration in the hedgehog $(\mathrm{HH})$ pathway. $\mathrm{HH}$ is one of several pathways that orchestrate embryogenesis by exerting partial and temporal control over proliferation, survival and cell-fate decisions. In response to paracrine signals, $\mathrm{HH}$ can completely inhibit a protein termed Patched-1 (PTCH-1), provoking the removal of its inhibition upon Smoothened, an enzyme involved in cell proliferation. As a result, $\mathrm{HH}$ hyperactivation is able to stimulate Smoothened, activating a cell-proliferation pathway, which, in normal cells, is silent, although it becomes hyperactive during embryogenesis. Uncontrolled $\mathrm{HH}$ signalling is sufficient to promote tumorigenesis in basal skin cells. Major genetic changes causing BCC include inactivating mutations of PTCH-1 and activating mutations of Smoothened (27,28). Vismodegib is a small molecule that is able to inhibit Smoothened, and is commonly used in the treatment of advanced BCCs $(29,30)$. A mounting body of evidence has demonstrated that, in a number of solid tumours, the ligand-dependent activation of $\mathrm{HH}$ signalling is potentiated through cross-talk with other critical molecular signalling pathways (31). Among these pathways are the Ras-RAF-mitogen-activated protein kinase kinase (MEK)-extracellular signal-regulated kinase (ERK) and the $\mathrm{PI} 3 \mathrm{~K} / \mathrm{Akt} / \mathrm{mammalian}$ target of rapamycin (mTOR) signalling pathways, and the EGFR and Notch signalling pathways. Thus, in NMSC, EGFR signalling acts synergistically with the $\mathrm{HH}$ pathway in the malignant transformation of cells (32).

On the basis of these findings, in the present review it is proposed that the hyperactivation of EGFR is a common feature in BCC and SCC, and that blockade of EGFR may be a therapeutic option for this category of tumours.

\section{Locally advanced NMSC: The role of cetuximab}

Standard options. Locally advanced NMSCs often are treated with Mohs surgery, and, when the risk of recurrence is high, with sentinel lymph-node dissection and, occasionally, with adjuvant radiotherapy or chemoradiation. Radiation or chemoradiation are employed only in patients not fit for surgery, due to disease extension, poor performance status or refusal of surgery. External beam radiation therapy for NMSC often consists of a three-dimensional conformal technique, using $6 \mathrm{MV}$ energy photon beams, with the aim of striking the deep parts of the tumour and lymph nodes. An additional dose of radiation, using electrons, is often administered, with the aim of an improved coverage of the external component of the tumour. Usually, 66-70 Gy, in fractions of $2 \mathrm{~Gy}$, are 
administered (5). Radiation therapy technology has evolved with improved efficacy and, at the same time, a reduction in the dose of radiation to the surrounding normal tissue. Intensity-modulated radiation therapy provides an improved dose conformation and uniformity, with the sparing of normal tissues, and an improved target-volume coverage and lower toxicity. Concomitant chemoradiation has been demonstrated to markedly prolong patient survival rates compared with the survival rates with radiation alone; the drug most often used in clinical trials has been cisplatin (6). However, cisplatin, which is administered at a dose of $100 \mathrm{mg} / \mathrm{m}^{2}$ of body surface for three weeks, is often associated with significant toxicity, in particular, nausea, vomiting and dysphagia. These toxic reactions frequently require discontinuation of the therapy, particularly in patients who are elderly ( $>75$ years old) or who have a poor performance status. Owing to its toxicity, cisplatin may require substitution with a better-tolerated drug.

Cetuximab. Given the paucity of non-resectable or metastatic cutaneous SCCs, reliable information on the frequency of the tumours' EGFR expression is limited. However, in a clinical trial enrolling patients affected by SCC, Toll et al (33) demonstrated that the expression of EGFR, as determined by immunostaining experiments, markedly correlated with EGFR gene amplification, assessed by fluorescence in situ hybridization. Additionally, the expression of EGFR in SCC was markedly higher when compared with that observed in actinic keratotic and normal skin cells. Similarly, EGFR gene amplification was identified in a markedly higher proportion of SCC, with respect to actinic keratosis.

Cetuximab is a chimaeric human/murine monoclonal antibody, which binds competitively to EGFR and prevents activation of the receptor, thus blocking activation of its downstream pathways (34). Compared with currently approved chemotherapies for skin carcinomas, cetuximab is better tolerated, the most frequently encountered side effects being those concerning the skin, namely acneiform eruption, xerosis, paronychia, hair changes, telangiectasia and hyperpigmentation. In clinical trials, cetuximab has been shown to be fairly efficacious for recurrent or metastatic chemo-refractory NMSC. Kalapurakal et al (35) treated eight patients, whose disease progressed following first-line platinum-containing chemotherapy with single-agent cetuximab. Of the patients, five achieved a complete remission and three obtained a partial remission, with an overall response rate of $100 \%$, although the duration of response was short: The disease in $63 \%$ of the patients progressed within six months.

The aforementioned study paved the way for further investigations of cetuximab in NMSC. Maubec et al (36) enrolled 36 patients with recurrent, metastatic inoperable BCC or SCC in a phase II trial. Cetuximab monotherapy was administered at a standard induction dose of $400 \mathrm{mg} / \mathrm{m}^{2}$ of body surface, followed by a weekly dose of $250 \mathrm{mg} / \mathrm{m}^{2}$ leading up to the progressive disease (PD) stage. A disease control rate (DCR), namely the sum of the complete response, partial response and stable disease at six weeks, was achieved in 25 patients $(69 \%)$, with an overall response rate of $28 \%$. Among the 31 evaluable patients, the development of an acneiform rash did not enable the prediction of a response to the treatment, although it did enable prediction of the mean progression-free survival and overall survival times. A number of case reports of cutaneous SCC patients treated with cetuximab also have been published, which have demonstrated that cetuximab may be a therapeutic option in patients with non-resectable cutaneous SCC $(37,38)$.

Concurrent cetuximab radiotherapy. Treatment of patients with NMSC, particularly those who are unsuitable for surgery, with cetuximab and exclusive radiation therapy may be an intriguing strategy. Preneau et al (39) performed a phase II study of cetuximab for non-resectable SCC. Among 20 patients enrolled, five were selected for treatment with radiotherapy (60-70 Gy) with concurrent cetuximab, and the remaining patients were treated with carboplatin-cetuximab or cetuximab alone. After two months, the responses were evaluated, and as a result, no patient was identified who had a complete remission. Of the five patients, four $(80 \%)$ had undergone a partial remission and the remaining patient (20\%) registered with stable disease, with a DCR of $100 \%$. The median progression-free survival was five months. Of the patients, four $(80 \%)$ experienced a serious adverse event (grade 3-4); in particular, in-field skin toxicity, namely an acneiform rash in the irradiated area, was the most frequent side effect. Patients selected to receive radiotherapy plus cetuximab had a higher response rate compared with those who received carboplatin with cetuximab or cetuximab alone (80 vs. 44 vs. $33 \%$, respectively).

Samstein et al (40) retrospectively analysed 12 patients treated with concurrent cetuximab-radiotherapy for locally advanced and non-resectable SCC. The patients were elderly: $75 \%$ had moderate to severe comorbidities, whereas $42 \%$ had immune dysfunction. Radiation therapy was delivered to all the patients via an intensity-modulated radiotherapy technique, reaching a median total dose of $60 \mathrm{~Gy}$; cetuximab was administered according to the standard weekly schedule. Complete and partial responses were noted in 36 and $27 \%$ of the patients, respectively, with an overall response rate of $64 \%$ and a DCR of $91 \%$. The median progression-free survival and overall survival times were 6.4 and 8.0 months, respectively. Considering the poor prognosis of the population, the response rate to this treatment was promising, although grade 2-3 adverse events were encountered in $83 \%$ of the patients: Skin rash was the most common, followed by fatigue, radiation dermatitis and infection. Almost $65 \%$ of the patients had side effects requiring hospitalization.

Helical tomotherapy represents a very important step in radiotherapeutic technical innovation, by allowing a further improvement in dose conformation and uniformity and the sparing of normal tissues. The association of helical tomotherapy and cetuximab has been documented in a case report (41). The patient was a 45-year-old woman, with a very advanced SCC arising from the sacral region and involving the spinal cord. A marked and durable response was observed on combining cetuximab with helical tomotherapy. Previously published data regarding this technique are few, although it is likely to deserve further investigation.

\section{Conclusion}

NMSCs are a very heterogeneous category of tumours, predominantly composed of $\mathrm{BCCs}$, which originate from the 
basal layer of the epidermis, and SCCs, which arise from keratinocytes in the superficial and corneous strata. BCCs have a low metastatic potential, although certain subtypes, particularly if poorly differentiated, may easily spread to the lymph nodes and distant sites. SCCs are more likely to be aggressive and invasive compared with BCCs, and the majority of locally advanced and advanced NMSCs are SCCs. Therapeutic strategies for locally advanced disease are similar for both diseases, and consist of surgery when possible, or surgery combined with chemoradiation. Little reliable information exists regarding the management of advanced NMSCs. Patients with advanced disease are relatively rare. Therefore, multi-institutional trials must be conducted to accrue adequate patient numbers. The literature primarily consists of isolated case reports and small case series. As discussed above, cisplatin-containing chemoradiotherapy is poorly manageable, and it may be substituted by cetuximab. At present, there is a lack of clinical trials assessing the efficacy of cetuximab in combination with radiation therapy in locally advanced NMSCs, and the majority of the trials are small, single-institution experiences, or case reports. One possible advance may be afforded by modification of the radiotherapy techniques, thereby allowing well-shaped irradiation, perfectly conformed on the target, and the coupling of radiotherapy with EGFR blockade. Nevertheless, skin toxicity observed in clinical trials has not been low.

Radiation dermatitis occurs in most patients receiving radiotherapy. Patients with SCC who receive radiotherapy in combination with EGFR inhibitors, including cetuximab, may develop a characteristic acne-like rash in addition to dermatitis. Radiation-induced keratinocyte damage induces DNA injury repair via activation of the p53 pathway and a simultaneous release of inflammatory cytokines as a consequence of the generation of free radicals, and, at the same time, keratinocytes demonstrate an increased expression of EGFR, possibly as a mechanism for repopulating irradiated areas (42). The use of EGFR inhibitors may be associated with the development of skin reactions, including a macular, papular, pustular rash, commonly referred to as acne-like rash, xerosis, fissures, telangiectasia and hair and nail changes (43). The pathophysiology of the skin reactions associated with EGFR inhibitors has yet to be fully elucidated, although the protein p27 may be involved, which is up-regulated on systemic cetuximab administration, leading to an impairment of the cell cycle, apoptosis and differentiation (44).

Several published clinical studies have been in favour of a multidisciplinary team management of these patients, with the joint aim of early recognition of any cutaneous side effects and administration of local and systemic drugs to resolve them (45). For example, the topical application of vitamin K3 (menadione), an EGFR phosphatase inhibitor, was shown to restore EGFR-mediated signalling in the skin, which had been altered by the administration of cetuximab (46).

Efficacy of the EGFR blockade has been observed for BCC and SCC: Gefitinib, a small molecule that inhibits EGFR, and panitumumab, a completely humanized monoclonal antibody directed against EGFR, have been demonstrated to have a certain amount of activity in $\operatorname{NMSC}(47,48)$. This last feature may depend on the biology of NMSC, since the EGFR pathway is often deregulated in SCC and in BCC. Notably, in $\mathrm{BCC}$, crosstalk between the $\mathrm{HH}$ pathway, which represents the predominantly disrupted pathway in this category of tumours, and the EGFR pathway has been identified (30).

A future approach may be to associate an $\mathrm{HH}$ pathway inhibitor, such as vismodegib, with cetuximab in the treatment of BCC, or to employ EGFR inhibition earlier in SCC. The only problem that arises is the variable frequency of ras mutations in NMSC, which, in certain previous studies, has reached $22 \%(24-26)$.

In conclusion, the association of radiation therapy and cetuximab, as is possible in the case of concomitant $\mathrm{HH}$ targeting (for BCC), should be taken into account for the treatment of locally advanced NMSC in the future. Skin toxicity, which is associated with the concomitant administration of cetuximab and radiotherapy, may be best treated by recourse to multidisciplinary team management, which may lead to earlier detection and an improved resolution of cutaneous side effects.

\section{References}

1. Rogers HW, Weinstock MA, Harris AR, Hinckley MR, Feldman SR, Fleischer AB and Coldiron BM: Incidence estimate of nonmelanoma skin cancer in the United States, 2006. Arch Dermatol 146: 283-287, 2010.

2. Christenson LJ, Borrowman TA, Vachon CM, Tollefson MM, Otley CC, Weaver AL and Roenigk RK: Incidence of basal cell and squamous cell carcinomas in a population younger than 40 years. JAMA 294: 681-690, 2005.

3. Chen JG, Fleischer AB Jr, Smith ED, Kancler C, Goldman ND, Williford PM and Feldman SR: Cost of nonmelanoma skin cancer treatment in the United States. Dermatol Surg 27: 1035-1038, 2001.

4. Rowe DE, Carroll RJ and Day CL Jr: Prognostic factors for local recurrence, metastasis and survival rates in squamous cell carcinoma of the skin, ear and lip. Implications for treatment modality selection. J Am Acad Dermatol 26: 976-990, 1992.

5. National Comprehensive Cancer Network Guidelines of Cancers by Site. Available online: http://www.ncen.org/professionals/physician. Accessed October 24, 2014.

6. Tanvetyanon T, Padhya T, McCaffrey J, Kish JA, Deconti RC, Trotti A and Rao NG: Postoperative concurrent chemotherapy and radiotherapy for high-risk cutaneous squamous cell carcinoma of the head and neck. Head Neck 37: 840-845, 2015

7. Bernier J, Domenge C, Ozsahin M, Matuszewska K, Lefèbvre JL, Greiner RH, Giralt J, Maingon P, Rolland F, Bolla M, et al: Postoperative irradiation with or without concomitant chemotherapy for locally advanced head and neck cancer. N Engl J Med 350: 1945-1952, 2004.

8. Jarkowski A III, Hare R, Loud P, Skitzki JJ, Kane JM III, May KS, Zeitouni NC, Nestico J, Vona KL, Groman A and Khushalani NI: Systemic therapy in advanced cutaneous squamous cell carcinoma (CSCC): The Roswell Park experience and a review of the literature. Am J Clin Oncol, 2014 (Epub ahead of print).

9. Mecca C, Ponzetti A, Caliendo V, Ciuffreda L and Lista P: Complete response of metastatic cutaneous squamous cell carcinoma to cetuximab plus paclitaxel. Eur J Dermatol 22: 758-761, 2012.

10. Nakamura K, Okuyama R, Saida T and Uhara H: Platinum and anthracycline therapy for advanced cutaneous squamous cell carcinoma. Int J Clin Oncol 18: 506-509, 2013.

11. DeConti RC: Chemotherapy of squamous cell carcinoma of the skin. Semin Oncol 39: 145-149, 2012.

12. Salomon DS, Brandt R, Ciardiello F and Normanno N: Epidermal growth factor-related peptides and their receptors in human malignancies. Crit Rev Oncol Hematol 19: 183-232, 1995.

13. Maubec E, Duvillard P, Velasco V, Crickx B and Avril MF: Immunohistochemical analysis of EGFR and HER-2 in patients with metastatic squamous cell carcinoma of the skin. Anticancer Res 25: 1205-1210, 2005.

14. Kalyankrishna S and Grandis JR: Epidermal growth factor receptor biology in head and neck cancer. J Clin Oncol 24: 2666-2672, 2006. 
15. Ciardiello F and Tortora G: A novel approach in the treatment of cancer: Targeting the epidermal growth factor receptor. Clin Cancer Res 7: 2958-2970, 2001.

16. Arteaga C: Targeting HER1/EGFR: A molecular approach to cancer therapy. Semin Oncol 30 (3 Suppl 7): 3-14, 2003.

17. Uribe P and Gonzalez S: Epidermal growth factor receptor (EGFR) and squamous cell carcinoma of the skin: Molecular bases for EGFR-targeted therapy. Pathol Res Pract 207: 337-342, 2011.

18. Kim S, Eleff M and Nicolaou N: Cetuximab as primary treatment for cutaneous squamous cell carcinoma to the neck. Head Neck 33 286-288. 2011.

19. Laimer K, Spizzo G, Gastl G, Obrist P, Brunhuber T, Fong D, Barbieri V, Jank S, Doppler W, Rasse M and Norer B: High EGFR expression predicts poor prognosis in patients with squamous cell carcinoma of the oral cavity and oropharynx: A TMA-based immunohistochemical analysis. Oral Oncol 43: 193-198, 2007.

20. Chang AR, Wu HG, Park CI, Jun YK and Kim CW: Expression of epidermal growth factor receptor and cyclin D1 in pretreatment biopsies as a predictive factor of radiotherapy efficacy in early glottic cancer. Head Neck 30: 852-857, 2008.

21. Kearsley JH, Furlong KL, Cooke RA and Waters MJ: An immunohistochemical assessment of cellular proliferation markers in head and neck squamous cell cancers. Br J Cancer 61: 821-827, 1990.

22. Bentzen SM, Atasoy BM, Daley FM, Dische S, Richman PI, Saunders MI, Trott KR and Wilson GD: Epidermal growth factor receptor expression in pretreatment biopsies from head and neck squamous cell carcinoma as a predictive factor for a benefit from accelerated radiation therapy in a randomized controlled trial. J Clin Oncol 23: 5560-5567, 2005.

23. Pedicini P, Nappi A, Strigari L, Jereczek-Fossa BA, Alterio D, Cremonesi M, Botta F, Vischioni B, Caivano R, Fiorentino A, et al: Correlation between EGFR expression and accelerated proliferation during radiotherapy of head and neck squamous cell carcinoma. Radiat Oncol 7: 143, 2012.

24. Pierceall WE, Goldberg LH, Tainsky MA, Mukhopadhyay T and Ananthaswamy HN: Ras gene mutation and amplification in human nonmelanoma skin cancers. Mol Carcinog 4: 196-202, 1991.

25. Khan SG, Mohan RR, Katiyar SK, Wood GS, Bickers DR, Mukhtar $\mathrm{H}$ and Agarwal R: Mutations in ras oncogenes: Rare events in ultraviolet $\mathrm{B}$ radiation-induced mouse skin tumorigenesis. Mol Carcinog 15: 96-103, 1996.

26. Mauerer A, Herschberger E, Dietmaier W, Landthaler M and Hafner C: Low incidence of EGFR and HRAS mutations in cutaneous squamous cell carcinomas of a German cohort. Exp Dermatol 20: 848-850, 2011.

27. Abidi A: Hedgehog signaling pathway: A novel target for cancer therapy: Vismodegib, a promising therapeutic option in treatment of basal cell carcinomas. Indian J Pharmacol 46: 3-12, 2014

28. Proctor AE, Thompson LA and O'Bryant CL: An inhibitor of the Hedgehog signaling pathway in the treatment of basal cell carcinoma. Ann Pharmacother 48: 99-106, 2014.

29. Tietze JK, Pfob M, Eggert M, von Preußen A, Mehraein Y, Ruzicka $\mathrm{T}$ and Herzinger T: A non-coding mutation in the 5 ' untranslated region of patched homologue 1 predisposes to basal cell carcinoma. Exp Dermatol 22: 834-835, 2013.

30. Ruiz Salas V, Alegre M, Garcés JR and Puig L: Locally advanced and metastatic basal cell carcinoma: Molecular pathways, treatment options and new targeted therapies. Expert Rev Anticancer Ther 14: 741-749, 2014.

31. Pandolfi S and Stecca B: Cooperative integration between HEDGEHOG-GLI signalling and other oncogenic pathways: Implications for cancer therapy. Expert Rev Mol Med 17: e5, 2015.

32. Brechbiel J, Miller-Moslin K and Adjei AA: Crosstalk between hedgehog and other signaling pathways as a basis for combination therapies in cancer. Cancer Treat Rev 40: 750-759, 2014.
33. Toll A, Salgado R, Yébenes M, Martín-Ezquerra G, Gilaberte M, Baró T, Solé F, Alameda F, Espinet B and Pujol RM: Epidermal growth factor receptor gene numerical aberrations are frequent events in actinic keratoses and invasive cutaneous squamous cell carcinomas. Exp Dermatol 19: 151-153, 2010

34. Perri F, Longo F, Ionna F and Caponigro F: Recent results of cetuximab use in the treatment of squamous cell carcinoma of the head and neck. Onco Targets Ther 2: 243-250, 2009.

35. Kalapurakal SJ, Malone J, Robbins KT, Buescher L, Godwin J and Rao K: Cetuximab in refractory skin cancer treatment. J Cancer 3: 257-261, 2012

36. Maubec E, Petrow P, Scheer-Senyarich I, Duvillard P, Lacroix L, Gelly J, Certain A, Duval X, Crickx B, Buffard V, et al: Phase II study of cetuximab as first-line single-drug therapy in patients with unresectable squamous cell carcinoma of the skin. J Clin Oncol 29: 3419-3426, 2011.

37. Bauman JE, Eaton KD and Martins RG: Treatment of recurrent squamous cell carcinoma of the skin with cetuximab. Arch Dermatol 143: 889-892, 2007.

38. Suen JK, Bressler L, Shord SS, Warso M and Villano JL: Cutaneous squamous cell carcinoma responding serially to single-agent cetuximab. Anticancer Drugs 18: 827-829, 2007.

39. Preneau S, Rio E, Brocard A, Peuvrel L, Nguyen JM, Quéreux G and Dreno B: Efficacy of cetuximab in the treatment of squamous cell carcinoma. J Dermatolog Treat 25: 424-427, 2014

40. Samstein RM, Ho AL, Lee NY and Barker CA: Locally advanced and unresectable cutaneous squamous cell carcinoma: Outcomes of concurrent cetuximab and radiotherapy. J Skin Cancer 2014: 284582, 2014

41. Falivene S, Giugliano FM, Grimaldi AM, Di Franco R, Toledo D, Muto M, Cammarota F, Borzillo V, Ascierto PA and Muto P: Tomotherapy concomitant with cetuximab, followed by cetuximab as single-agent therapy for unresectable squamous cell carcinoma of the skin: A case report. BMC Dermatol 14: 15 , 2014.

42. Muller K and Meineke V: Radiation-induced alterations in cytokine production by skin cells. Exp Hematol 35 (4 Suppl 1): S96-S104, 2007.

43. Segaert S and Van Cutsem E: Clinical signs, pathophysiology and management of skin toxicity during therapy with epidermal growth factor receptor inhibitors. Ann Oncol 16: 1425-1433, 2005.

44. Busam KJ, Capodieci P, Motzer R, Kiehn T, Phelan D and Halpern AC: Cutaneous side-effects in cancer patients treated with the antiepidermal growth factor receptor antibody $\mathrm{C} 225$. Br J Dermatol 144: 1169-1176, 2001.

45. Perez-Soler R, Zou Y, Li T and Ling YH: The phosphatase inhibitor menadione (vitamin K3) protects cells from EGFR inhibition by erlotinib and cetuximab. Clin Cancer Res 17: 6766-6777, 2011

46. Bernier J, Bonner J, Vermorken JB, Bensadoun RJ, Dummer R, Giralt J, Kornek G, Hartley A, Mesia R, Robert C, et al: Consensus guidelines for the management of radiation dermatitis and coexisting acne-like rash in patients receiving radiotherapy plus EGFR inhibitors for the treatment of squamous cell carcinoma of the head and neck. Ann Oncol 19: 142-149, 2008.

47. Foote MC, McGrath M, Guminski A, Hughes BG, Meakin J, Thomson D, Zarate D, Simpson F and Porceddu SV: Phase II study of single-agent panitumumab in patients with incurable cutaneous squamous cell carcinoma. Ann Oncol 25: 2047-2052, 2014.

48. Perez CA, Song H, Raez LE, Agulnik M, Grushko TA, Dekker A, Stenson K, Blair EA, Olopade OI, Seiwert TY, et al: Phase II study of gefitinib adaptive dose escalation to skin toxicity in recurrent or metastatic squamous cell carcinoma of the head and neck. Oral Oncol 48: 887-892, 2012. 